\title{
Editorial
}

\section{Carbon-Based Nanomaterials as Novel Nanosensors}

\author{
Qin Hu, ${ }^{1}$ Evan K. Wujcik, ${ }^{2,3,4}$ Antonios Kelarakis, ${ }^{5}$ Jobin Cyriac, ${ }^{6}$ and Xiaojuan Gong ${ }^{7}$ \\ ${ }^{1}$ Department of Chemistry, The State University of New York at Buffalo, Buffalo, NY, USA \\ ${ }^{2}$ Materials Engineering and Nanosensor [MEAN] Laboratory, Department of Chemical and Biological Engineering, \\ The University of Alabama, Tuscaloosa, AL, USA \\ ${ }^{3}$ Department of Materials Science, The University of Alabama, Tuscaloosa, AL, USA \\ ${ }^{4}$ Center for Materials for Information Technology [MINT], The University of Alabama, Tuscaloosa, AL, USA \\ ${ }^{5}$ School of Physical Sciences and Computing, University of Central Lancashire, Lancashire, UK \\ ${ }^{6}$ Department of Chemistry, Indian Institute of Space Science and Technology, Thiruvananthapuram, India \\ ${ }^{7}$ Institute of Environmental Science, and School of Chemistry and Chemical Engineering, Shanxi University, Taiyuan, China
}

Correspondence should be addressed to Qin Hu; qinhu@buffalo.edu

Received 4 June 2017; Accepted 5 June 2017; Published 5 November 2017

Copyright (c) 2017 Qin Hu et al. This is an open access article distributed under the Creative Commons Attribution License, which permits unrestricted use, distribution, and reproduction in any medium, provided the original work is properly cited.

In recent years, nanosensor technology has experienced a rapid development because of the extensive scientific efforts in understanding of nanoscale phenomena and achieving innovative nanofabrication techniques. Carbon-based nanomaterials (CBNs), such as fullerenes, graphene, nanodiamonds, carbon nanotubes, and carbon nanodots, have recently gained considerable attention among scientific communities due to their unique chemical and physical properties. Thanks to intensive research efforts, the CBNs have found their place in a wide range of applications. These CBNs stand out as novel nanosensors due to their supreme performance in detecting heavy metal ions, gas molecules, food additives, antibodies, and toxic pesticides, as well as reporters for bioimaging.

This special issue, to be published in 2017, addresses recent progress in the synthesis, characterization, structureproperty relationships and applications of CBNs as novel nanosensors. We are confident that the accrual of these contributions will facilitate the applications of CBNs as innovative nanosensors in meeting the urgent needs for environmental monitoring, food safety control, healthcare, homeland security, and so forth. We have selected five papers, representing four different frontiers of this topic: graphene, silver nanoparticles, carbon nanotubes, and carbon nanodots.

J. Wang et al. reported the preparation of graphene oxide/cellulose nanofibril hybrid aerogel for adsorptive removal of four kinds of antibiotics, achieving the removal percentages of $81.5 \%, 79.5 \%, 79.1 \%$, and $73.9 \%$ for doxycycline, chlortetracycline, oxytetracycline, and tetracycline, respectively. S. Xiong et al. reported the preparation of a leaflike $\mathrm{BiVO}_{4}$-reduced graphene oxide composite $\left(\mathrm{BiVO}_{4}-\mathrm{rGO}\right)$ and demonstrated that the $\mathrm{BiVO}_{4}$-rGO exhibited higher photocatalytic capacity towards the degradation of rhodamine $B$ dye under visible-light irradiation compared with pure $\mathrm{BiVO}_{4}$.

$\mathrm{H}$. Younes et al. studied the effect of saline solution on the electrical response of single wall carbon nanotubes-epoxy nanocomposites and found that a drop of saline solution on the surface of the nanocomposites film increases the resistance by $50 \%$.

L. Yumei et al. reported a rapid biosynthesis method of silver nanoparticles based on flocculation and reduction of an exopolysaccharide from Arthrobacter sp. B4 and investigated its antimicrobial activity and phytotoxicity.

Finally, Q. Hu et al. presented a comprehensive review on characterization and analytical separation of fluorescent carbon nanodots, which summarized the most commonly used techniques for the characterization and analytical separation of carbon nanodots and concludes with an outlook towards the future development in this area.

As editors of this special issue, we would like to express our gratitude to all the authors for their valuable high-quality contributions. We hope that the readers will find interesting information in the carefully selected and thoroughly 
reviewed articles. We look forward greatly to their future success.

Qin $H u$

Evan K. Wujcik Antonios Kelarakis

Jobin Cyriac

Xiaojuan Gong 

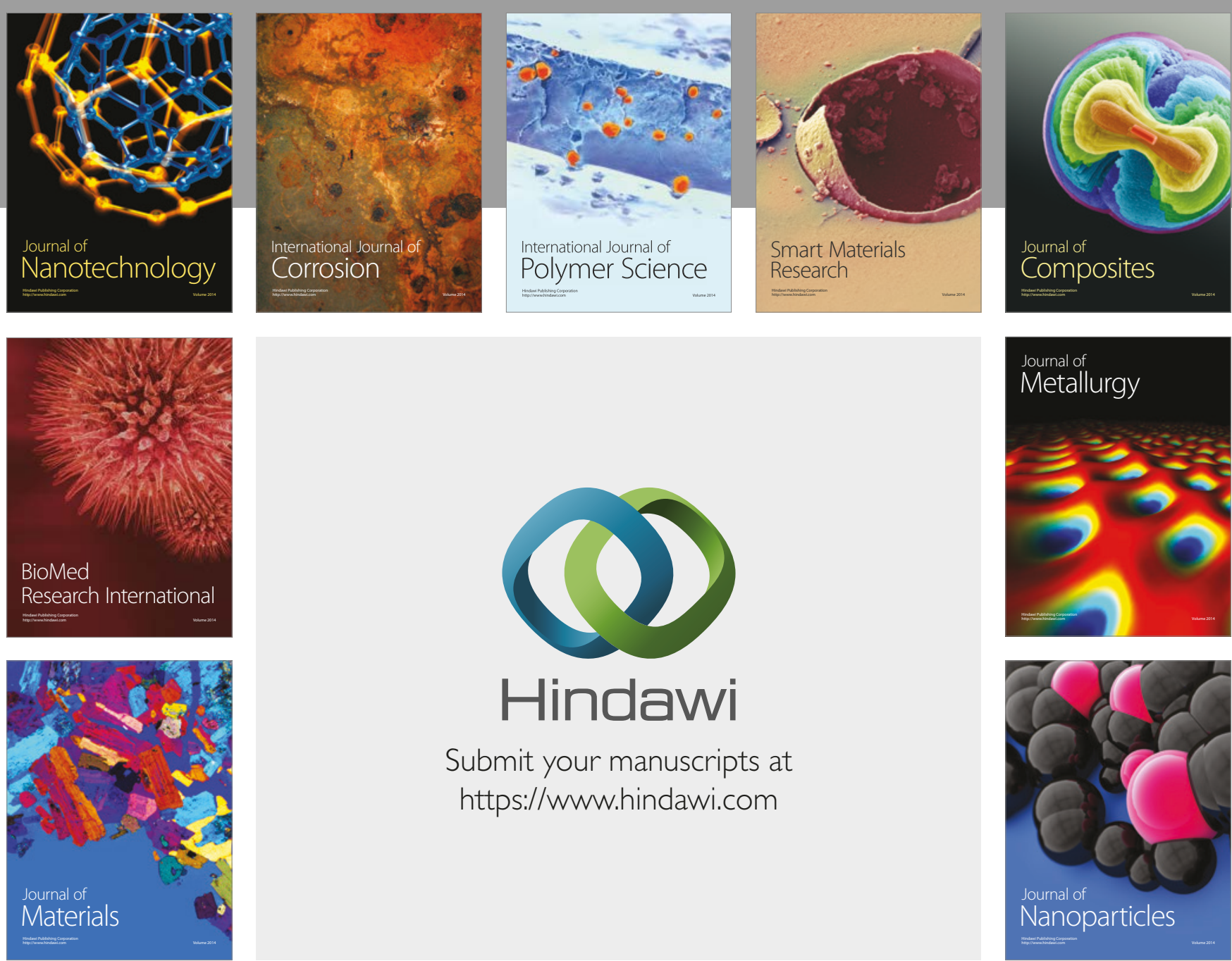

\section{Hindawi}

Submit your manuscripts at

https://www.hindawi.com
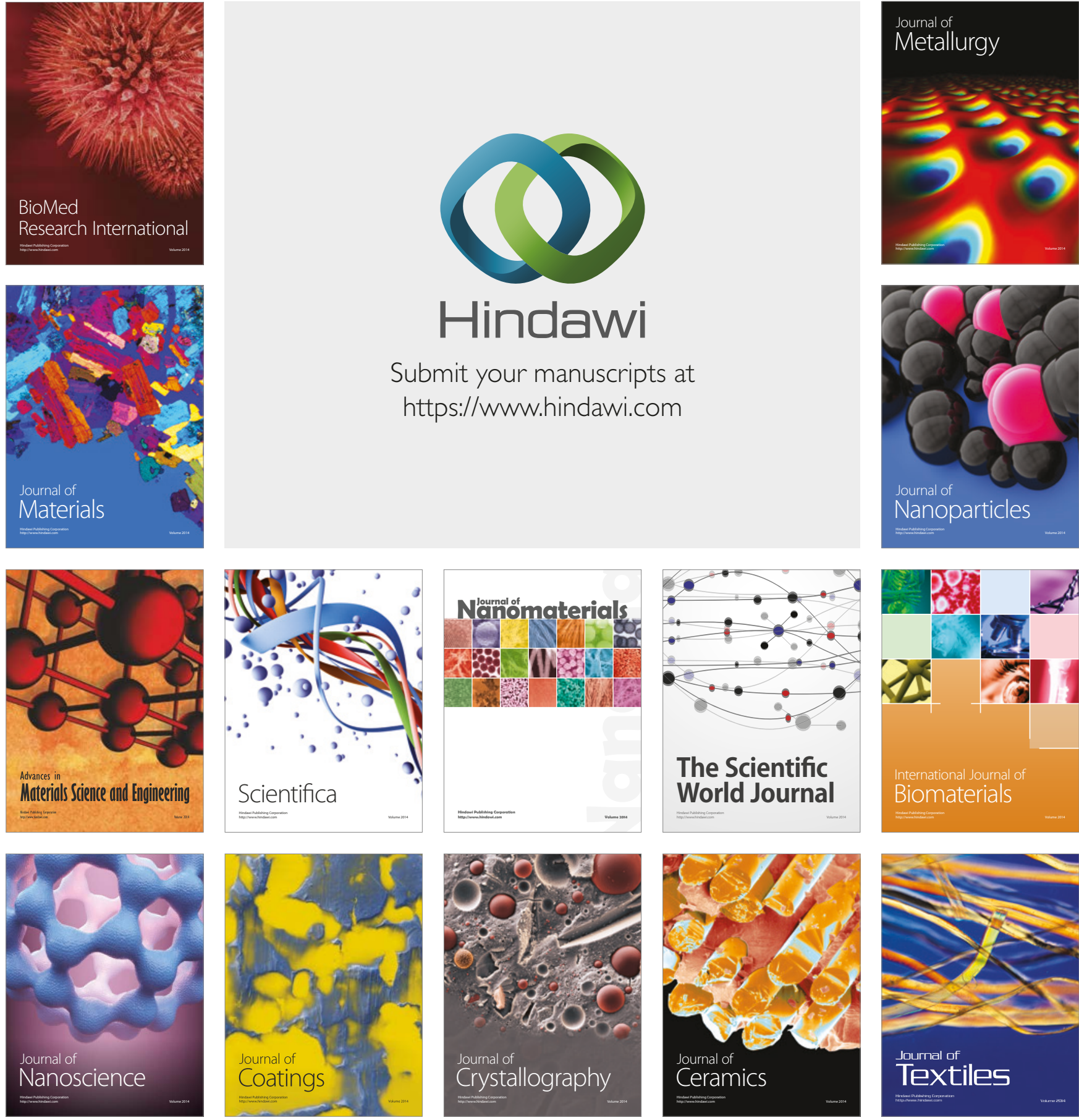

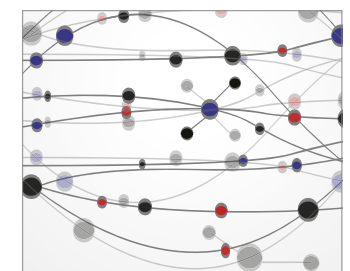

The Scientific World Journal
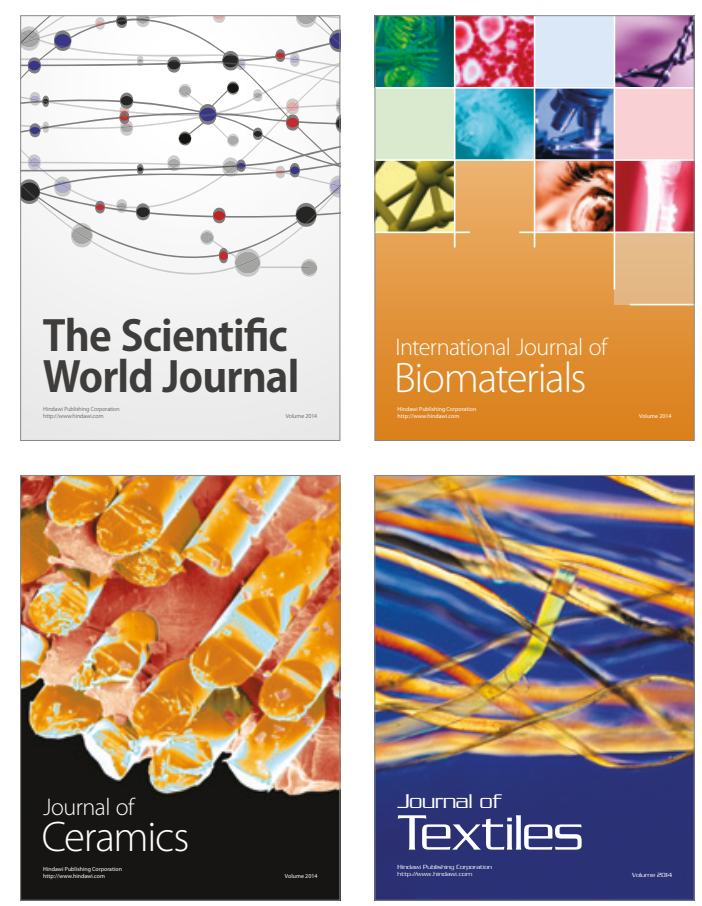